\title{
The importance of apnea in patients undergoing angiography of thoracic vessels - a protocol for acute pulmonary thromboembolism research
}

Importância da apneia em pacientes submetidos à angiotomografia de vasos torácicos protocolo para pesquisa de tromboembolismo pulmonar agudo

Kátia Ayumi Takeda ${ }^{1}$, Walther Yoshiharu Ishikawa ${ }^{1}$, Camila dos Santos Silva $^{1}$, Fábio Augusto ${ }^{1}$, Elaine Ferreira da Silva ${ }^{1}$, Adriano Tachibana ${ }^{1}$, Marcelo Buarque de Gusmão Funari ${ }^{1}$

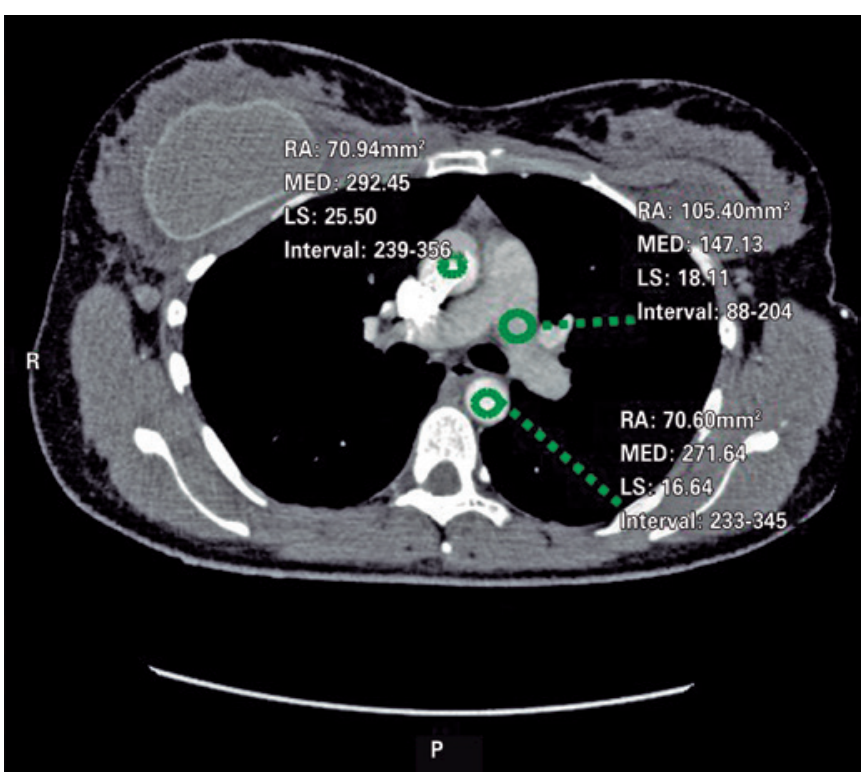

Figure 1. Contrast agent density in pulmonary artery of 147.13 , contrast agent density in ascending aorta of 292.45 , and contrast agent density in descending aorta of 271.64

This was 37-year-old woman, 10 days after surgery, who underwent breast prosthesis replacement, abdominoplasty and liposuction. Upon admission, she was hemodynamically stable, febrile and reported constant pain on left hemithorax region, but without dyspnea.

An angio-CT of thoracic vessels was carried out. During intravenous injection of contrast agent, the

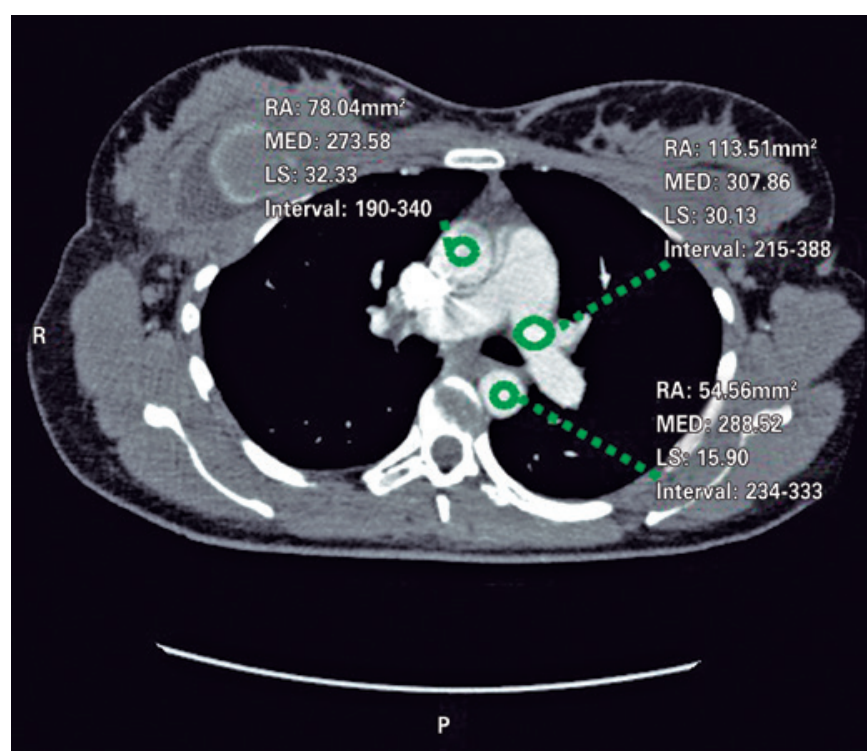

Figure 2. Contrast agent density in pulmonary artery of 307.86 , contrast agent in ascending aorta of 273.58 and contrast agent of descending aorta of 288.52

patient was anxious and started crying during the exam. We believe that, a Valsalva maneuver was performed on inspiration apnea requested during the exam therefore causing an intrathoracic pressure that resulted only in a thoracic aortic contrast (Figure 1). A new contrast agent was intravenously injected after apnea orientation, and the Valsalva maneuver was not requested, which resulted in

\footnotetext{
${ }^{1}$ Hospital Israelita Albert Einstein, São Paulo, SP, Brazil.

Corresponding author: Kátia Ayumi Takeda - Avenida Albert Einstein, 627/701 - Morumbi - Zip code: 05652-900 - São Paulo, SP, Brazil - Phone: (55 11) 98213-3025 - E-mail: katia.takeda@einstein.br Received on: Oct 20, 2016 - Accepted on: Feb 17, 2017
} 


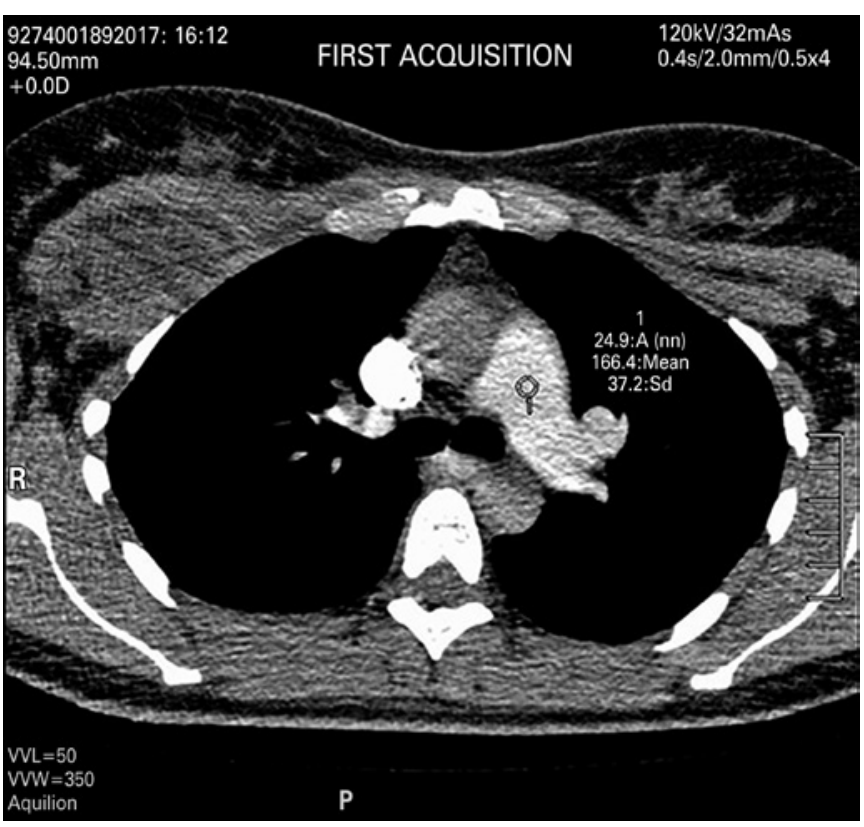

Figure 3. Contrast agent density in pulmonary artery of 166.4 for acquisition of exam sequence

precisely diagnostic images (Figure 2). Two acquisitions were carried out using manual trigger when peak of pulmonary artery contrast occurred (Figure 3 and 4).

The diagnosis of acute pulmonary thromboembolism (PTE) is based on clinical probability, use of D-dimer dosage and imaging assessment - including the angioCT because it is a rapid, non-invasive procedure with high sensibility and specificity $(83 \%$ and $96 \%$, respectively). ${ }^{(1)}$ This method enables to evaluate all area of mediastinum and pulmonary parenchyma, and, the use of intravenous iodine contrast agent in its maximal peak enables to evaluate the pulmonary artery and its distal branches and thoracic aorta.

Studies show that negative angio-CT exam, even in case of good quality images, is enough to exclude PTE. $^{(2,3)}$ An important factor that affects negatively the quality of exam is the transitory interruption of contrast agent, which was first described by Gosselin et al., as a physiologic artifact, ${ }^{(4)}$ which also entails low contrast of pulmonary artery and its segments. This vascular phenomena must occur when patient undergo a short time deep inhaling before image acquisitions,

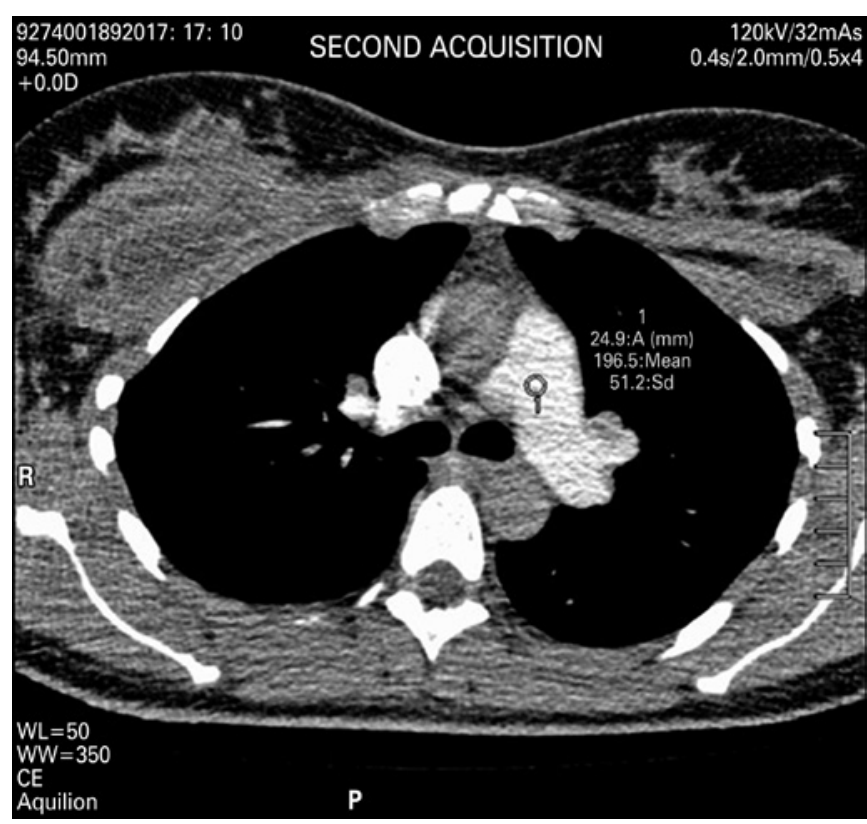

Figure 4. Contrast agent density in pulmonary artery of 196.5 for acquisition of second sequence

which result in an increase of blood venous return to the inferior vena cava, or reduction in contrast agent flow reduction by the superior vena cava. The non-opacified blood in left atrium dilutes the contrast agent of the superior vena cava, ${ }^{(5)}$ therefore causing low attenuation of pulmonary artery and difficult to diagnose PTE.

\section{REFERENCES}

1. Duru $S$, Keleşoğlu A, Ardiç $S$. Clinical update on pulmonar embolism. Arch Med Sci. 2014;10(3):557-65.

2. Goodman LR, Lipchick RJ, Kuzo RS, Liu Y, McAuliffe TL, O'Brien D. Subsequent pulmonary embolism: risck after a negative helical CT pulmonary angiogram-prospective comparison with scintigraphy. Radiology. 2000;215(2):535-42.

3. Swensen SJ, Sheedy PF 2nd, Ryu JH, Pickett DD, Schelek CD, Ilstrup DM, et al. Outcomes after withholding anticoagulation from patients with suspected acute pulmonary embolism and negative computed tomography findings: a cohort study. Mayo Clin Proc. 2002;77(2):130-8

4. Gosselin MV, Rassner UA, Thieszen SL, Phillips J, Oki A. Contrast dynamics during CT pulmonary angiogram: analysis of an inspiration associated artifact. J Thorac Imaging. 2004;19(1):1-7.

5. Bernabé-García JM, García-Espasa C, Arenas-Jiménez J, Sánchez-Payá J, de la Hoz-Rosa J, Carreres-Polo JO. Has "respiratory coaching" before deep inspiration an impact on the incidence of transient contrast interruption during pulmonary CT angiography? Insights Imaging. 2012;3(5):505-11. 